\title{
Penile Strangulation by Metallic Rings
}

\author{
Mohammad Rashid Farooqui $\cdot$ R. S. Meena
}

Received: 27 April 2010 /Accepted: 24 February 2011 /Published online: 7 August 2011

(C) Association of Surgeons of India 2011

\begin{abstract}
A case of penile strangulation in a 35 year old male truck driver by profession reported here. Two metallic rings were self introduced upto the base of penis, in order to prevent spontaneous ejaculation at night. There was marked oedema of penis distal to rings, and these rings were removed with an indigenous technique, non-operatively
\end{abstract}

Keywords Penile strangulation $\cdot$ Metallic rings

\section{Introduction}

Penile strangulation by various objects (e.g., wedding rings, rubber bands, plumbing metal ring, plastic bottle neck, steel bearing, etc.) [1-3] presents a situation that challenges the surgeon to be innovative in selecting the treatment.

Here, we report a case in which a healthy male, with normal mental state, had put two metaflic rings of stainless steel over his penis, and thereafter presented to the emergency with an extremely swollen penis, as he himself was unable to remove the rings.

M. R. Farooqui

Department of Medicine, Government Medical College,

Kota, Rajasthan, India

R. S. Meena

Department of Surgery, Government Medical College,

Kota, Rajasthan, India

M. R. Farooqui ( $\square)$

3rd Floor, 19/337 Nisha Nasheman, Near Pyaga Near Pyaga

School, Soorarajpol gate, Shreepura,

Kota, Rajasthan PIN 324006, India

e-mail: dr_mrfarooqui@rediffmail.com

\section{Case Report}

A 35-year-old man presented to M.B.S. Hospital, Kota, Rajasthan with an extremely swollen penis. On examination, gross oedema was found on the distal part of the penis, and two metal rings were found applied to the base of the penis,(Fig. 1), which could not be taken out.

On taking detailed history, it was revealed that he was advised by his friend to pass a metallic ring over the base of the penis, to obviate penile erection and autoejaculation at night. He was practicing this method successfully for the past 1 year. The patient was shifted to emergency O.T. in an effort to remove the metallic rings. Lidocaine jelly was applied over the penis, multiple punctures were given and compression was applied on the penis by hand, due to which thin streams of fluid came out from the puncture sites, in spite of all the efforts the rings could not be removed, and no suitable instrument was available to cut the rings safely. Finally, an intravenous drip set tube was applied circumferentially as a tourniquet, starting from the tip of the penis to its base to have an even compression (Fig. 2). The compression was maintained for $4 \mathrm{~min}$, and this procedure was repeated five times (in between the tourniquet application the penile compression was maintained by squeezing the penis with palm). This manoeuver reduced penile oedema dramatically and metallic rings could be retrieved manually over a lubricated jelly. The whole procedure took around $50 \mathrm{~min}$. The two rings were of $3.5 \mathrm{~cm} \times 2.5 \mathrm{~cm} \times 2 \mathrm{~cm}$ and $3 \mathrm{~cm} \times 2 \mathrm{~cm} \times 1.5 \mathrm{~cm}$ external diameter, internal diameter and width, respectively (Fig. 3).

There was no damage to the penis at the strangulation site as well as on the distal part. Penis was cleaned with povidone-iodine solution, an antibiotic ointment was 


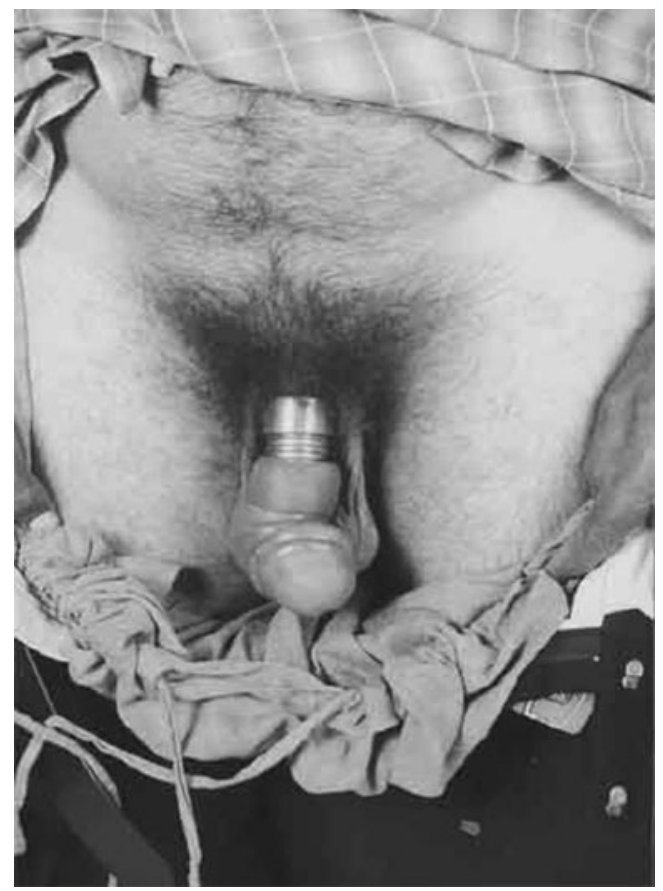

Fig. 1 Swellened penis with 2 mettalic rings applied to base

applied and dressed with Vaseline gauze and light pressure bandage was applied.

The patient remained cooperative during the procedure. Before starting the procedure the patient was premedicated with atropine $0.6 \mathrm{mg}$ injection, midazolam $5 \mathrm{mg}$ and tramadol $\mathrm{HCl} 100 \mathrm{mg}$ were administered intravenously with a patency IV drip and $2 \%$ lidocaine jelly was applied

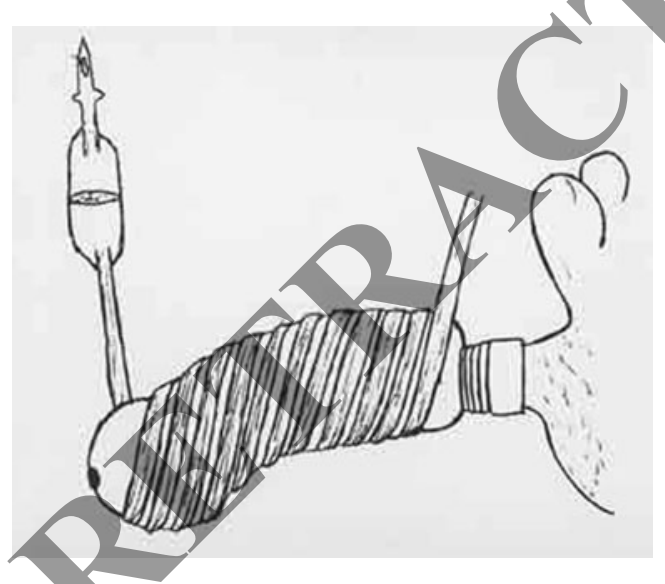

Fig. 2 Separated metallic rings with measurement

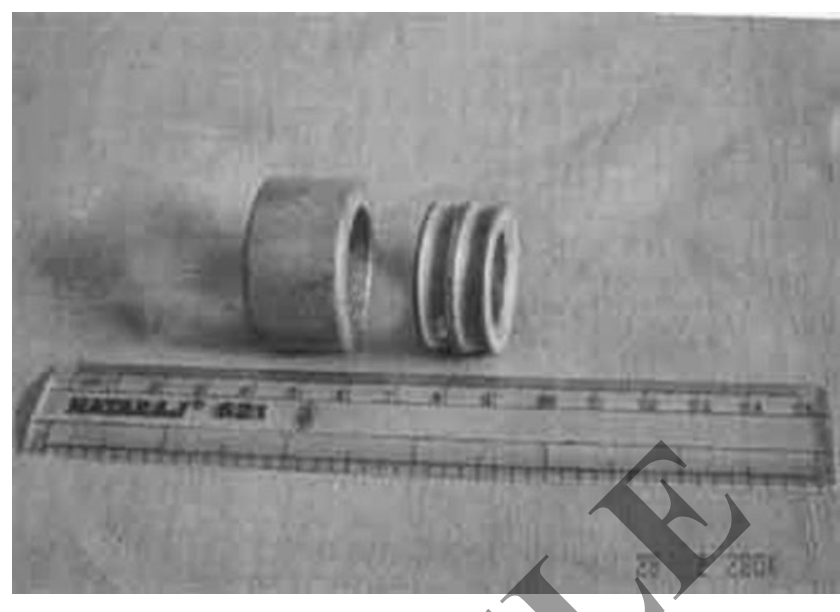

Fig. 3 Intravenous drip set method to reduce edema

over the penis. Antibiotics and tetanus prophylaxis was also given. Later, psychoanalysis revealed no abnormality.

\section{Comment}

Incidence of penile strangulation injuries is not frequent, yet such types of injuries are most difficult to deal with. Increased oedema distal to occlusion restricts venous and lymphatic flow. Eventually the constriction may impede arterial supply and may result in gangrene, which may require amputation of penis. There is no standard protocol mentioned to deal with such cases. Every case needs individual approach depending on the circumstances and facilities available. The method described above is highly effective, cost saving, complication free and can be done by any practitioner.

\section{References}

1. McLaughlin T, Coyner W (1989) Removal of a strangulating metal bearing from the penis. J Urol 141(3):617

2. Perabo FG, Steiner G, Albers P, Müller SC (2002) Treatment of penile strangulation caused by constrictions devices. Urology 59 (1): 137

3. Kadioğlu A, Cayan S, Ozcan F, Tellaloğlu S (1995) Treatment of penile incarceration in an impotent patient. Int Urol Nephrol 27 (5):639-641 